\title{
Nutrition, Genes and Modern Disease: A Current Dilemma or a Legacy of our Past
}

Stephen Myers ${ }^{1^{*}}$ and Sheridan Williamson ${ }^{2}$

${ }^{1}$ Federation University Australia, Mount Helen Campus, Ballarat, Victoria 3350, Australia

${ }^{2}$ University of the Sunshine Coast, Sippy Downs, Sunshine Coast, Queensland, 4556, Australia

*Corresponding author: Stephen Myers, Federation University Australia, Mount Helen Campus, Ballarat, Victoria 3350 , Australia, Tel: +61 400 718 920; E-mail: smyers@federation.edu.au

Rec date: May 17, 2014, Acc date: June 18, 2014, Pub date: June 26, 2014

Copyright: ( 2014 Myers S, et al. This is an open-access article distributed under the terms of the Creative Commons Attribution License, which permits unrestricted use, distribution, and reproduction in any medium, provided the original author and source are credited.

\begin{abstract}
Contemporary humans are genetically adapted to the environment that their ancestors survived in and that consequently selected their genetic makeup. Since the agricultural revolution some 10,000 years ago, the lifestyles and dietary requirements of modern humans have changed dramatically. It is suggested that these changes have occurred too recently on an evolutionary time scale for the modern human genome to adapt. Therefore, our ancestral genome is ill-suited for our current modern consumption and existence, and thus contributes to diseases associated with contemporary lifestyles, such as cardiovascular disease, obesity and type 2 diabetes. It is therefore suggested that a diet similar to our ancestors could circumvent many of our modern illnesses and serve as a reference for better nutrition, health, and longevity. Although this model should certainly be commended for its simplistic dietary practices that no doubt improve health and well-being; its premise is cemented in the thrifty genome hypothesis and the fact that humans are modern hunters and gatherers whose genome is ill-suited for modern diets. This is a disjointed view of modern humans and our ability to evolve under different eco regions and nutritional pressures through post-genomic and post-transcriptional changes in our genome. Accordingly, a major challenge associated with nutritional research is to understand how these changes in our genome reflect on our nutrition habits and lifestyles to ameliorate many of our modern lifestyle diseases.
\end{abstract}

Keywords: Paleolithic; Hunters and gatherers; Type 2 diabetes; Thrifty genome; Epigenetics

\section{Introduction}

The survival of Homo sapiens throughout evolution depended on a number of critical factors. These included the procurement of shelter and food and the ability to adapt to a dynamic and unpredictable environment through gradual changes in the genome over many generations $[1,2]$. In this context, humans have evolved as highlydeveloped Hunters and Gatherers (HG) beginning more than 2 million years ago and continuing to about 10,000 years ago; a time which heralded the dawn of agriculture [3]. Since the beginning of the agricultural revolution (and thus less than 1\% of Homo evolutionary time) it is suggested that the human genome has remained essentially unchanged $[4,5]$. It is proposed that during the Paleolithic era, the feeding habits of HG would have been challenged by punctuated times of famine and feast, leading to the assumption that humans traits were selected for during the Paleolithic period [6]. Accordingly, since the agricultural revolution, our principle metabolic and physiological processes have not had the evolutionary time to adapt to the major dietary changes introduced during this period [3-5]. Thus, the traits of our ancestors are outpaced by cultural change and therefore modern humans exist in an environment for which the HG genome is ill-suited [2]. This discordance between our ancestral genetics and the advent of changes in nutritional activity associated with a contemporary lifestyle could be responsible for many of the so called "civilized" disorders associated with insulin resistance including obesity, cardiovascular disease and diabetes. It is therefore proposed that a Paleolithic diet characterized by higher protein levels, less total fat, more essential fatty acids, and higher fibre could serve as a reference standard for modern human nutrition because it is increasingly acknowledged that low rates of cardiovascular disease and other related disorders such as type 2 diabetes have been observed among more contemporary HG [6,7].

\section{Insulin Resistance and Our "Primitive" Genome}

Over five decades ago, Neel [7] suggested that insulin resistance evolved as an adaptive trait to help survive in primitive times during cycles of famine and feast. In fact, the ability to specifically modulate metabolic responses to insulin is evolutionarily conserved in insects, worms, and vertebrates, including humans [8]. Neel noted "during the first 99 per cent or more of man's life on earth, while he existed as a hunter and gatherer, it was often feast or famine" [7]. Accordingly, carriers of this "thrifty" genotype would have a selective advantage over less 'thrifty' genotypes. Neel proposed that a 'thrifty' genotype would be "exceptionally efficient in the intake and/or utilization of food" [7]. Consequently, during times of famine, the 'thrifty' genotype would allow for increased fat storage and thus represent a survival advantage over a less 'thrifty' genotype [2]. While this thrifty genotype would have been beneficial during times of regular famine, it would also pose a liability in modern westernized societies that have regular access to food sources [9]. Accordingly, sedentary lifestyles and positive caloric imbalances through the selection of highly processed foods rich in carbohydrates, are suggested to be implicated in a discordance between our ancestral genes and modern circumstances leading to the dysregulation of ancestral homeostatic processes and contributing to modern diseases such as obesity and type 2 diabetes $[1,2,7,10]$. 
Support for this hypothesis comes from several reports; for example studies with diabetic Australian aboriginal peoples noted an improvement in glucose tolerance, insulin sensitivity and the blood lipid profile with a relatively short ( 7 weeks) implementation of the traditional HG diet of beef, kangaroo, fish, turtle, vegetables and honey: $50 \%$ protein, $40 \%$ fat, and $10 \%$ carbohydrate [11]. Similarly, in a clinical trial on normal healthy sedentary subjects that consumed a Paleolithic diet (lean meat, fruit, vegetables and nuts) for 10 days, there was an observable improvement in blood pressure, a decrease in insulin secretion, an increase in insulin sensitivity, and an improvement in blood lipid profiles [3]. Equally, in a randomized cross-over study of three months duration, diabetic patients receiving a Paleolithic diet (lean meat, fish, fruit, vegetables, root vegetables, eggs, and nuts) showed a reduction in body weight, BMI and waist circumference, lower $\mathrm{HbAlc}$, TAG and diastolic blood pressure, and an improvement in glycaemic control [12]. These studies are further supported by Lindeberg et al. [13] who reported that patients with ischemic heart disease concomitant with glucose intolerance or type 2 diabetes had a large improvement in insulin sensitivity and glucose tolerance when receiving a Paleolithic diet. Even in non-human studies the Paleolithic diet has been successfully used as a model to explain modern disease. For example, pigs fed a cereal-based swine feed or cereal-free Paleolithic diet consisting of vegetables, fruit, meat and tubers, conferred higher insulin sensitivity, lower C-reactive protein and lower blood pressure when compared to pigs on the cereal-based feed alone [14].

\section{Our Dynamic Genome}

While these kinds of studies in humans have heralded a major paradigm shift by incorporating a decidedly evolutionary interpretation of contemporary diet and health, and the rise in incidence and prevalence in obesity and diabetes, the evolutionary discordance model assumes that our current genome has hardly changed from that of our ancestral genome [6]. Although we can argue about exactly how our ancestors ate and lived, it is clear that the time we have spent in agrarian settlements rather than as HG is miniscule within the span of human evolution. In Paleofantasy, biologist Marlene Zuk [15] suggests that there is a strong body of evidence that our genome has gone through many changes since humans spread out across the globe and developed agriculture and therefore it is difficult to determine a single way of eating to which we were, and remain, best suited. For example, Thompson et al. [16] observed large allele frequency differences between African, Americans and non-African populations and distance from the equator for a variant of the CYP3A5 gene, designated CYP3A $5^{\star} 1{ }^{*} 3$. The CYP3A subfamily of cytochrome P450 genes catalyzes the metabolism of endogenous substrates that include bile acids, steroids, and environmental carcinogens such as pesticides [16,17]. It was suggested that this rare variant CYP $3 A 5^{\star} 11^{\star} 3$ is implicated in salt and water retention and a risk for salt-sensitive hypertension [18]. Interestingly, the frequency of the CYP $3 \mathrm{~A} 5^{\star} 1 /{ }^{\star} 3$ allele increased with distance from the equator with the lowest frequency in sub-Saharan Africa, and highest in European and East Asia populations [18].

Human populations use a variety of subsistent strategies to exploit a broad range of ecoregions and dietary needs, and most certainly there is a wide physiological and morphologic variation among populations, some of which is most undoubtedly modified by genetic adaptations to local environments and dietary requirements $[19,20]$. In this context, Hancock et al. [21] tested the idea that cold and heat stress exerted strong selective pressures on the biological processes underlying common metabolic disorders. They examined the patterns of genetic variance in 82 candidate genes for common metabolic disorders across 52 globally dispersed populations in order to understand if climate played a role in shaping genotypic variation. These studies identified several generic Single Nucleotide Polymorphisms (SNPs) that had significant spatial patterns with climate variation. For example, signals of spatially varying selection for nonsynonymous SNPs were found at the Fatty Acid Binding protein 2 (FABP2) gene. The derived allele at SNP rs1799883, which increases strongly with latitude, was found to increase affinity for long-chain fatty acids, and is consistent with its role in protection against cold temperatures by increasing BMI and fuel for heat generation [21]. In addition, this SNP is also associated with insulin resistance, increased fasting insulin concentration, fasting fatty acid oxidation, and reduced glucose uptake [22,23].

Similarly, in a large genome-wide scan for signals associated with very recent positive selection Voight et al. (2006)[20] it was noted that there were strong signals of selection in the Alcohol Dehydrogenase $(\mathrm{ADH})$ cluster in East Asians. Moreover, genes involved in carbohydrate metabolism such as mannose metabolism (MAN2A1 in Yoruba and East Asians), sucrose (SI in East Asians), and lactose (LCT in Europeans); and genes implicated in the metabolism of dietary fatty acids including long-chain fatty acid transport protein 4 and peroxisome proliferator-activated receptor delta (SLC27A4 and PPARD in Europeans), carnitine/acylcarnitine translocase (SLC25A20 in East Asians), nuclear receptor coactivator 1 (NCOA1 in Yoruba), and leptin receptor (LEPR in East Asians) also showed strong signals of selection.

Other more recent examples of genetic adaptation due to dietary specialisation include variations in the lactase gene, a trait that is believed to be advantageous in agro-pastoral populations where milk is a major staple of the adult diet, and variations in the amylase gene in agricultural populations that rely on high starch diets as their major staple [24,25]. Similarly, Hancock et al. [19] provided evidence for several SNPs that show concordant differences in allele frequencies across populations that live in the same geographic region, but that differ in their ecoregions, dietary status, or mode of subsistence. These authors identified several strong associations with polar ecoregions, with foraging, and diets rich in roots and tubers. Moreover, several of the strongest signals overlap with those implicated in energy metabolism, and include SNPs associated with influencing glucose levels and susceptibility to type 2 diabetes. Furthermore, several changes in pathways associated with starch and sucrose metabolism were enriched which suggests adaptations to a diet rich in roots and tubers. Among the genes with strong signals in these pathways were several involved in glycogen synthesis and degradation, and liver enzyme hydrolyzes ( $\beta$-D-glucoside and $\beta$-D-galactoside) which may be involved in the detoxification of plant glycosides, such as those found in roots and tubers. The polar ecoregions displayed changes in pathways associated with energy metabolism such as pyruvate metabolism, glycolysis, and gluconeogenesis and suggests a link between adaptations to cold tolerance/stress and energy metabolism.

These studies have reasonable experimental power to detect recent adaptations and it is therefore feasible that most of the genetic signals coincide with the transition to agriculture and animal farming [26]. One mechanism in which natural selection might act on selection specificity to diet for example could be through the targeting of specific gene regulatory components such as promoters, enhancers, etc. Consistent with this notion, Haygood et al. (2007) [27] compared 
the rates of evolution along the human lineage between a promoter region and nearby intronic sequences. They compared the rates of nucleotide substitutions in the promoter and intronic sequences between human and chimpanzees and identified several cis-regulatory regions of genes known to be involved in neural development and in nutrition, particularly glucose metabolism, evolved at faster rates than introns. They therefore suggest that human adaptations, particularly to changes in diet, have ascended primarily through cis-regulatory regions [27].

In summary, it appears that we may not be at the mercy of our ancestral genetic makeup, and therefore it may be possible to overcome our genetic destiny by implementing specific life style choices. Accordingly, in a rapidly changing environment, it is suggested that the adaptation of a DNA sequence to specific changes is a slow process [28]. Thus, the flexibility for rapid adaptation to the environment is therefore facilitated by mechanisms that allow for the modification of a phenotype by epigenetic programming [28].

\section{Gene-Nutrient Interactions: Epigenetic Programming}

Our human diet has undergone profound changes in the last five decades and this trend is likely to continue and become more complex well into the 21st century. The nutrient composition of the human diet varies between populations and geographical regions and ethnicity plays a large role in the type of food sources that are consumed [29]. There is a growing awareness of the importance and impact of diet and nutrition on health, but there is little understanding on how these changes affect our genetic 'make-up' and contribute to disease processes.

Primarily, there are three types of nutrient-gene interactions: direct interactions, genetic variations, and epigenetic interactions [29]. Direct interactions typically involve a nutrient that interacts with a receptor usually a transcription factor that is implicated in modulating gene transcription. A classic example of this is the Liver X Receptor (LXR) family of nuclear receptors that are activated by cholesterol and bile acids and facilitate reverse cholesterol efflux and lipogenesis [30,31]. Genetic variations include SNPs that are known to influence nutrient requirements. For example, functional SNPs in the inflammatory genes TNFA, IL-1 and IL- 6 have been reported to interact with dietary fatty acids to regulate the production and secretion of cytokines, predisposing an individual to inflammation and altering obesity $[16,17]$. Epigenetic interactions involve nutrients that can alter the structure of DNA (or of histone proteins in chromatin) so that gene expression is chronically altered. Studies have successfully demonstrated effects on DNA methylation of alcohol, the B vitamins, protein, and micronutrients [29]. In this context, epigenetic changes facilitated by dietary nutrients are emerging as a critical determinant of an organism's response to its environment and biological functionality and disease susceptibility [25].

Epigenetics describes the characteristics of heritable changes in gene function that occur independently of changes in the DNAs nucleotide structure or sequence [32]. At the most basic level, epigenetics is about information that is present in the genome, independent to that coded in the DNA sequence of nucleotides [29]. Epigenetic change may be directly influenced by diet leading to changes in health status, or 'programming' the epigenome so that it responds to nutrients throughout the lifetime of the organism. Moreover, it has recently been demonstrated that the epigenome is highly dynamic and changes in response to aging, physical exercise, and nutrients for example
[29,32]. Epigenetics is emerging as perhaps the most important mechanism whereby the diet and nutrients can directly affect the genome and thus the modulation of gene expression [28].

Epigenetics is also under the influence of time and the way in which processes can impact on the epigenome to result in epigenetic 'footprints' that can persist for variable amounts of time and thus, influence future metabolic function and health outcomes [29,33]. For example, in one landmark study, the recruitment of individuals who were prenatally exposed to famine during the Dutch Hunger Winter (1944-1945) had, six decades later, epigenetic changes in the IGF2 gene (a key factor in growth and development) when compared to their unexposed, same sex, siblings [34]. The association was specific for preiconceptional exposure, and thus, strengthens the hypothesis that early development is a crucial period for establishing and maintaining epigenetic marks [34]. Thus, nutritional factors at critical stages in life can result in relatively stable epigenetic marks that persist over decades and therefore affect health outcomes, not only for the individual, but also through heritable developmental epigenetic changes during pregnancy to the offspring. For example, global DNA methylation analysis has detected regions of the DNA that are highly variable in methylation status in human subjects, some of which were consistently associated with BMI over time [35].

Accordingly, extensive human epidemiological data have indicated that prenatal and early postnatal nutrition can influence adult susceptibility to diet related diseases including obesity, cancer, heart disease and type 2 diabetes [33,34,36]. For example, studies on seasonally occurring diets in women from rural Gambia identified seasonal variations in methyl-donor nutrients around the time of conception that influence several plasma biomarkers that predict increased/decreased methylation at metastable epialleles (MEs) [37]. Moreover, maternal BMI was also predicative of systemic infant DNA methylation at MEs, and is significant in the perspective of identifying potential markers associated with offspring adiposity and changes in energy homeostasis. Developmental programming such as in these examples is thought to be an evolutionary advantage for the offspring genome to be optimally programmed in response to the maternal environment before birth so that it is metabolically prepared for its new environment after birth [29]. This concept suggests that the early life environment influences offspring characteristics later in life, including the predisposition to develop diseases associated with metabolism [38]. Moreover, to add another level of complexity, it is known that individuals can respond differently to the same dietary intake. For example, dietary cholesterol can cause changes in plasma cholesterol that is dependent on the individual [39-41].

Dietary factors can also alter the post-translational modification of histones and thus alter chromatin structure to influence gene transcription. Zhang et al. (2012) [42] showed that, in rats when fed a high fat diet, some developed obesity while others displayed an obesity-resistant phenotype. These authors demonstrated induction of senescence and aging pathways in obese rats via p16INK4a and p21Cip1 is associated with histone modifications (acetylation and methylation). Similarly, the offspring from rats fed a low protein diet during gestation showed a decrease in the expression of the cell cycle regulator p21 in the mammary gland which was associated with a histone modification at the promoter of the p21 gene [43]. These authors suggest that a maternal protein-restricted diet during pregnancy may alter cell cycle control in offspring and thereby predisposing them to the risk of developing breast cancer. 
Accordingly, nutrition's greatest challenge will be to establish basic protocols and principles that are amendable to improving health in all individuals, with the goal of maintaining good health and prevention of disease. Epigenetics will no doubt provide some of the answers to these challenges; however, nongenomic factors are also intimately involved in an individual's response to nutrients, their health status, and risk of disease [34]. Clearly, nutrition during both the pre- and early post-natal life can programme persistent changes in health and vitality into adulthood. Therefore, understanding the impact of diet on the post-genomic and post-transcriptional processes, taking into account heritable epigenetic traits, are needed to understand personalized diet and ongoing health and longevity.

\section{Conclusion}

It has become increasingly popular among many professional communities that consuming a diet similar to our ancestors has enormous therapeutic health benefits over that of more westernized diets consisting predominately of highly refined foods. It is suggested that the human genome has not evolved since the agricultural revolution and therefore, diseases associated with modern lifestyle changes such as cardiovascular disease, obesity and type 2 diabetes is concomitant with our ancestor genome being ill-suited to these modern nutritional processes. While modern disease is undoubtedly associated with poor nutrition habits and reduced physical activity, it is unlikely that thrifty genes are the only explanation for this phenomenon. In fact, we now know that the genome has continued to evolve, and at times, rapidly, in response to environmental and nutritional pressures through genomic SNPs and epigenetic programming. While the evolutionary thrifty gene model has provided a valuable framework for understanding human nutrition in the context of evolution, the assumption that modern culture has outstripped our ancestral genes and therefore is the fundamental cause of westernized disease has resulted in a fragmented view of the our ability to adapt to different nutritional pressures and ecoregions and how our dietary patterns can lead to dynamic changes in the genome that affect the health status, of not only our offspring, but well into our adult life many decades later.

\section{References}

1. Chakravarthy MV, Booth FW (2004) Eating, exercise, and "thrifty" genotypes: connecting the dots toward an evolutionary understanding of modern chronic diseases. J Appl Physiol (1985) 96: 3-10.

2. Zimmet P, Thomas CR (2003) Genotype, obesity and cardiovascular disease--has technical and social advancement outstripped evolution? J Intern Med 254: 114-125.

3. Frassetto LA, Schloetter M, Mietus-Synder M, Morris RC Jr, Sebastian A (2009) Metabolic and physiologic improvements from consuming a paleolithic, hunter-gatherer type diet. Eur J Clin Nutr 63: 947-955.

4. Eaton SB (2006) The ancestral human diet: what was it and should it be a paradigm for contemporary nutrition? Proc Nutr Soc 65: 1-6.

5. Mann N (2007) Meat in the human diet: An anthropological perspective. Nutrition \& Dietetics 64: S102-S107.

6. Turner BL, Thompson AL (2013) Beyond the Paleolithic prescription: incorporating diversity and flexibility in the study of human diet evolution. Nutr Rev 71: 501-510.

7. NEEL JV (1962) Diabetes mellitus: a "thrifty" genotype rendered detrimental by "progress"? Am J Hum Genet 14: 353-362.

8. Katic M, Kahn CR (2005) The role of insulin and IGF-1 signaling in longevity. Cell Mol Life Sci 62: 320-343.
9. Stöger R (2008) The thrifty epigenotype: an acquired and heritable predisposition for obesity and diabetes? Bioessays 30: 156-166.

10. Eaton SB, Konner M (1985) Paleolithic nutrition. A consideration of its nature and current implications. N Engl J Med 312: 283-289.

11. O'Dea K (1984) Marked improvement in carbohydrate and lipid metabolism in diabetic Australian aborigines after temporary reversion to traditional lifestyle. Diabetes 33: 596-603.

12. Jönsson T, Granfeldt Y, Ahrén B, Branell UC, Pålsson G, et al. (2009) Beneficial effects of a Paleolithic diet on cardiovascular risk factors in type 2 diabetes: a randomized cross-over pilot study. Cardiovasc Diabetol $8: 35$.

13. Lindeberg S, Jönsson T, Granfeldt Y, Borgstrand E, Soffman J, et al. (2007) A Palaeolithic diet improves glucose tolerance more than a Mediterranean-like diet in individuals with ischaemic heart disease. Diabetologia 50: 1795-1807.

14. Jonsson T, Ahren B, Pacini G, Sundler F, Wierup N, et al. (2006) A Paleolithic diet confers higher insulin sensitivity, lower C-reactive protein and lower blood pressure than a cereal-based diet in domestic pigs. Nutrition \& Metabolism 3: 39.

15. Zuk M (2013). Paleofantasy: what evoltion really tells us about sex, diet, and how we live, (W.W. Norton \& Company, Inc, New York. ISBN: 978-0-393-347-92-0).

16. Yu Z, Han S, Cao X, Zhu C, Wang X, et al. (2012) Genetic polymorphisms in adipokine genes and the risk of obesity: a systematic review and meta-analysis. Obesity (Silver Spring) 20: 396-406.

17. Joffe YT, Collins M, Goedecke JH (2013) The relationship between dietary fatty acids and inflammatory genes on the obese phenotype and serum lipids. Nutrients 5: 1672-1705.

18. Thompson EE, Kuttab-Boulos H, Witonsky D, Yang L, Roe BA, et al. (2004) CYP3A variation and the evolution of salt-sensitivity variants. Am J Hum Genet 75: 1059-1069.

19. Hancock AM, Witonsky DB, Ehler E, Alkorta-Aranburu G, Beall C, et al. (2010) Colloquium paper: human adaptations to diet, subsistence, and ecoregion are due to subtle shifts in allele frequency. Proc Natl Acad Sci U S A 107 Suppl 2: 8924-8930.

20. Voight BF, Kudaravalli S, Wen X, Pritchard JK (2006) A map of recent positive selection in the human genome. PLoS Biol 4: e72.

21. Hancock AM, Witonsky DB, Gordon AS, Eshel G, Pritchard JK, et al. (2008) Adaptations to climate in candidate genes for common metabolic disorders. PLoS Genet 4: e32.

22. Baier LJ, Sacchettini JC, Knowler WC, Eads J, Paolisso G, et al. (1995) An amino acid substitution in the human intestinal fatty acid binding protein is associated with increased fatty acid binding, increased fat oxidation, and insulin resistance. J Clin Invest 95: 1281-1287.

23. Albala B C, Jiménez R B, Pérez B F, Liberman G C (2006) [Fatty acid binding protein 2 (FABP-2) polymorphism, obesity and insulin resistance]. Rev Med Chil 134: 372-379.

24. Hancock AM, Alkorta-Aranburu G, Witonsky DB, Di Rienzo A (2010) Adaptations to new environments in humans: the role of subtle allele frequency shifts. Philos Trans R Soc Lond B Biol Sci 365: 2459-2468.

25. Perry GH, Dominy NJ, Claw KG, Lee AS, Fiegler H, et al. (2007) Diet and the evolution of human amylase gene copy number variation. Nat Genet 39: 1256-1260.

26. Luca F, Perry GH, Di Rienzo A (2010) Evolutionary adaptations to dietary changes. Annu Rev Nutr 30: 291-314.

27. Haygood R, Fedrigo O, Hanson B, Yokoyama KD, Wray GA (2007) Promoter regions of many neural- and nutrition-related genes have experienced positive selection during human evolution. Nat Genet 39: 1140-1144.

28. Siddique RA, Tandon M, Ambwani T, Rai SN, Atreja SK (2009) Nutrigenomics: Nutrient-Gene Interactions. Food Reviews International 25: 326-345.

29. Haggarty P (2013) Epigenetic consequences of a changing human diet. Proc Nutr Soc 72: 363-371. 
Citation: Myers S, Williamson S (2014) Nutrition, Genes and Modern Disease: A Current Dilemma or a Legacy of our Past. J Diabetes Metab 5: 393. doi:10.4172/2155-6156.1000393

Page 5 of 5

30. Myers SA, Wang S-CM, Muscat GEO (2006) The Chicken Ovalbumin Upstream Promoter-Transcription Factors Modulate Genes and Pathways Involved in Skeletal Muscle Cell Metabolism. Journal of Biological Chemistry 281: 24149-24160.

31. Huang C (2014) Natural modulators of liver X receptors. J Integr Med 12 : 76-85.

32. Franks PW, Ling C (2010) Epigenetics and obesity: the devil is in the details. BMC Med 8: 88 .

33. Waterland RA, Jirtle RL (2004) Early nutrition, epigenetic changes at transposons and imprinted genes, and enhanced susceptibility to adult chronic diseases. Nutrition 20: 63-68.

34. Heijmans BT, Tobi EW, Stein AD, Putter H, Blauw GJ, et al. (2008) Persistent epigenetic differences associated with prenatal exposure to famine in humans. Proc Natl Acad Sci U S A 105: 17046-17049.

35. Feinberg AP, Irizarry RA, Fradin D, Aryee MJ, Murakami P, et al. (2010) Personalized epigenomic signatures that are stable over time and covary with body mass index. Sci Transl Med 2: 49ra67.

36. Fernandez-Twinn DS, Ozanne SE (2010) Early life nutrition and metabolic programming. Ann N Y Acad Sci 1212: 78-96.

37. Dominguez-Salas P, Moore SE, Baker MS, Bergen AW, Cox SE, et al. (2014) Maternal nutrition at conception modulates DNA methylation of human metastable epialleles. Nature communications 5: 3746.
38. Aiken CE, Ozanne SE (2014) Transgenerational developmental programming. Hum Reprod Update 20: 63-75.

39. German JB, Zivkovic AM, Dallas DC, Smilowitz JT (2011) Nutrigenomics and personalized diets: What will they mean for food? Annu Rev Food Sci Technol 2: 97-123.

40. Turner PR, Konarska R, Revill J, Masana L, La Ville A, et al. (1984) Metabolic study of variation in plasma cholesterol level in normal men. Lancet 2: 663-665.

41. Cohen JC, Kiss RS, Pertsemlidis A, Marcel YL, McPherson R, et al. (2004) Multiple rare alleles contribute to low plasma levels of HDL cholesterol. Science 305: 869-872.

42. Zhang X, Zhou D, Strakovsky R, Zhang Y, Pan Y-X (2012) Hepatic cellular senescence pathway genes are induced through histone modifications in a diet-induced obese rat model. American Journal of Physiology - Gastrointestinal and Liver Physiology 302: G558-G564.

43. Zheng S, Rollet M, Yang K, Pan YX (2012) A gestational low-protein diet represses p21(WAF1/Cip1) expression in the mammary gland of offspring rats through promoter histone modifications. Br J Nutr 108: 998-1007. 